\title{
Bioinformatics analysis of fibroblasts exposed to TGF- $\beta$ at the early proliferation phase of wound repair
}

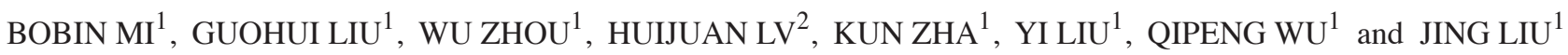 \\ ${ }^{1}$ Department of Orthopaedics, Union Hospital, Tongji Medical College, Huazhong University of Science \\ and Technology, Wuhan, Hubei 430022; ${ }^{2}$ Department of Rheumatology, Tangdu Hospital, \\ The Fourth Military Medical University, Xi'an, Shaanxi 710000, P.R. China
}

Received January 14, 2017; Accepted August 4, 2017

DOI: $10.3892 / \mathrm{mmr} .2017 .7619$

\begin{abstract}
The aim of the current study was to identify gene signatures during the early proliferation stage of wound repair and the effect of TGF- $\beta$ on fibroblasts and reveal their potential mechanisms. The gene expression profiles of GSE79621 and GSE27165 were obtained from GEO database. Differentially expressed genes (DEGs) were identified using Morpheus and co-expressed DEGs were selected using Venn Diagram. Gene ontology (GO) function and Kyoto Encyclopedia of Genes and Genomes (KEGG) pathway enrichment analysis of DEGs were performed using the Database for Annotation, Visualization and Integrated Discovery (DAVID) online tool. Protein-protein interaction (PPI) networks of the DEGs were constructed using Cytoscape software. PPI interaction network was divided into subnetworks using the MCODE algorithm and the function of the top one module was analyzed using DAVID. The results revealed that upregulated DEGs were significantly enriched in biological process, including the Arp2/3 complex-mediated actin nucleation, positive regulation of hyaluronan cable assembly, purine nucleobase biosynthetic process, de novo inosine monophosphate biosynthetic process, positive regulation of epithelial cell proliferation, whereas the downregulated DEGs were enriched in the regulation of blood pressure, negative regulation of cell proliferation, ossification, negative regulation of gene expression and type I interferon signaling pathway. KEGG pathway analysis showed that the upregulated DEGs were enriched in shigellosis, pathogenic Escherichia coli infection, the mitogen-activated protein kinase signaling pathway, Ras signaling pathway and bacterial invasion of epithelial cells. The downregulated DEGs were
\end{abstract}

Correspondence to: Dr Guohui Liu, Department of Orthopaedics, Union Hospital, Tongji Medical College, Huazhong University of Science and Technology, 1277 Jiefang Avenue, Wuhan, Hubei 430022, P.R. China

E-mail: liuguohui@medmail.com.cn

Key words: bioinformatics analysis, fibroblasts, transforming growth factor- $\beta$, wound repair enriched in systemic lupus erythematosus, lysosome, arachidonic acid metabolism, thyroid cancer and allograft rejection. The top 10 hub genes were identified from the PPI network. The top module analysis revealed that the included genes were involved in ion channel, neuroactive ligand-receptor interaction pathway, purine metabolism and intestinal immune network for IgA production pathway. The functional analysis revealed that TGF- $\beta$ may promote fibroblast migration and proliferation and defend against microorganisms at the early proliferation stage of wound repair. Furthermore, these results may provide references for chronic wound repair.

\section{Introduction}

Fibroblasts are widely distributed throughout the mesenchyme where they synthesize extracellular matrix (ECM) proteins that form a structural framework to support tissue architecture and function in steady-state conditions (1). Skin wound repair is a complex process that involves inflammation, proliferation and a remolding phase. At the early proliferation stage, fibroblast migration to the wound site is important for the formation of provisional ECM, such as collagen and fibronectin, in which the respective cell migration and organization takes place in successful repair (2-22).

Various signaling pathways, the transforming growth factor- $\beta$ (TGF- $\beta$ )/SMAD pathway in particular, have been investigated in the process of wound healing; however, the mechanisms of these pathways remain to be elucidated. TGF- $\beta$, a multifunctional cytokine secreted by macrophages, and its effect on wounds is controversial $(23,24)$. TGF- $\beta$ may stimulate collagen production in dermal fibroblasts by fibroblast-to-myofibroblast transition (25), the excess amount of TGF- $\beta$ increases collagen deposition, resulting in a keloid (26). TGF- $\beta$ that is produced during inflammatory phase by macrophages is an important mediator of fibroblast activation and tissue repair (27). Although the effect of TGF- $\beta$ on wounds has been previously identified, the complete underlying effect of TGF- $\beta$ on fibroblasts at the early proliferation phase of wound repair remain poorly understood.

Microarray technology has been used to obtain information on the genetic alteration that occurs during many diseases (28-30). The current study used bioinformatics to identify the differentially expressed genes (DEGs) of fibroblasts 
treated with TGF- $\beta$ for $24 \mathrm{~h}$. Then, the gene ontology (GO) and pathway enrichment were analyzed. By analyzing their biological function and pathway, we may determine the effect of TGF- $\beta$ at the early stage of wound repair.

\section{Materials and methods}

Microarray data. Gene expression omnibus (GEO; www.ncbi. nlm.nih.gov/geo) contains original submitter-supplied records and curated DataSets, which is freely available to users. Two gene expression profiles (GSE79621 and GSE27165) were obtained from the GEO database. Data of fibroblast samples and TGF- $\beta 24 \mathrm{~h}$ treated fibroblasts samples from the two gene expression profiles. The GSE79621 contained 3 fibroblast samples and 3TGF- $\beta 24 \mathrm{~h}$-treated fibroblast samples. The GSE27165 contained 2 fibroblast samples and 2 TGF- $\beta$ 24 h-treated fibroblast samples.

Identification of DEGs. The data was processed using web-based tool Morpheus (https://software.broadinstitute .org/morpheus/), which is a matrix visualization and analysis platform designed to support visual data exploration. The expressions of mRNAs with Signal to noise $>1$ or signal to noise $<1$ was defined as DEGs. The co-expressed upregulated and downregulated DEGs of the two gene expression profiles were identified with a Venn Diagram (http://bioinfogp.cnb. csic.es/tools/venny/index.html; Venny 2.1.0).

GO and Kyoto Encyclopedia of Genes and Genomes (KEGG) pathway enrichment analysis. The common upregulated and downregulated DEGs were analyzed using Database for Annotation, Visualization and Integrated Discovery version 6.7 (DAVID; david.ncifcrf.gov), an online program that provides a comprehensive set of function annotation tools for researchers to understand the biological meaning lists of genes. In order to analyze the DEGs at the function level, GO enrichment and KEGG pathway analyses were performed using DAVID. $\mathrm{P}<0.05$ was considered to indicate statistically significant difference.

Protein-protein interaction (PPI) network analysis. To further understand the functional interactions between these DEGs a PPI network was used. The DEGs were mapped with the Search Tool for the Retrieval of Interacting Genes (STRING; www.string-db.org) and experimentally validated interactions with a combined score of $>0.5$ were selected as significant. Then, the PPI network was constructed and visualized using Cytoscape software (version 3.4.0). The top 10 essential nodes ranked by degree were selected. The plug-in Molecular Complex Detection (MCODE) was used to identify the modules of the PPI network in Cytoscape. The criteria were set as follows: MCODE score $\geq 4$ and number of nodes $>4$. The function enrichment analysis of DEGs in the top module was performed using DAVID.

\section{Results}

Identification of DEGs. A total number of samples analyzed were 5 fibroblast samples and 5 TGF- $\beta 24$ h-treated fibroblast samples. The gene expression profiles were analyzed

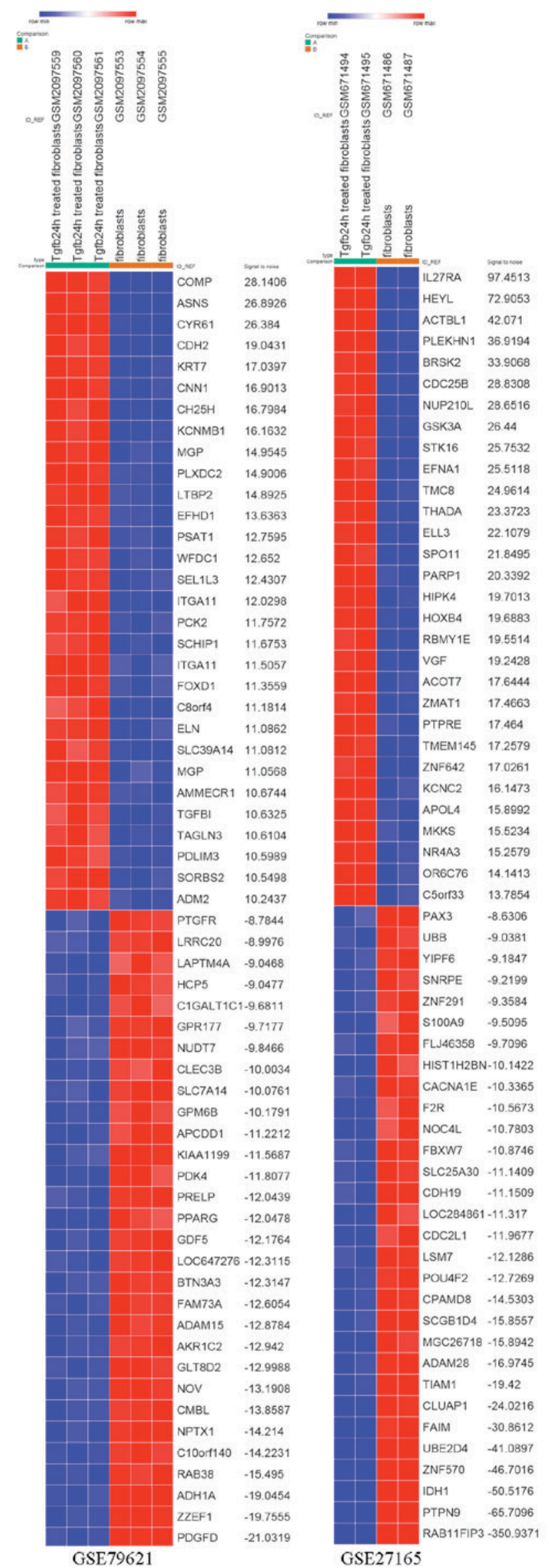

Figure 1. Heat map of the top 60 differentially expressed genes of GSE79621 and GSE27165 (30 upregulated and 30 downregulated). Red, upregulation; blue, downregulation. 
Table I. GO analysis of upregulated and downregulated differentially expressed genes in biological processes.

A, Upregulated

\begin{tabular}{llrr}
\hline Term & \multicolumn{1}{c}{ Function } & Count & P-value \\
\hline GO:0034314 & Arp2/3 complex-mediated actin nucleation & 5 & $6.98 \times 10^{-4}$ \\
GO:1900106 & Positive regulation of hyaluranon cable assembly & 3 & 0.001053124 \\
GO:0009113 & Purine nucleobase biosynthetic process & 3 & 0.003423324 \\
GO:0006189 & De novo IMP biosynthetic process & 3 & 0.005071039 \\
GO:0050679 & Positive regulation of epithelial cell proliferation & 6 & 0.006688708 \\
& & & \\
\hline
\end{tabular}

B, Downregulated

\begin{tabular}{|c|c|c|c|}
\hline Term & Function & Count & P-value \\
\hline GO:0008217 & Regulation of blood pressure & 9 & $5.66 \times 10^{-5}$ \\
\hline GO:0008285 & Negative regulation of cell proliferation & 18 & 0.00333738 \\
\hline GO:0001503 & Ossification & 7 & 0.005354786 \\
\hline GO:0010629 & Negative regulation of gene expression & 9 & 0.006461428 \\
\hline GO:0060337 & Type I interferon signaling pathway & 6 & 0.00863459 \\
\hline
\end{tabular}

GO, gene ontology.
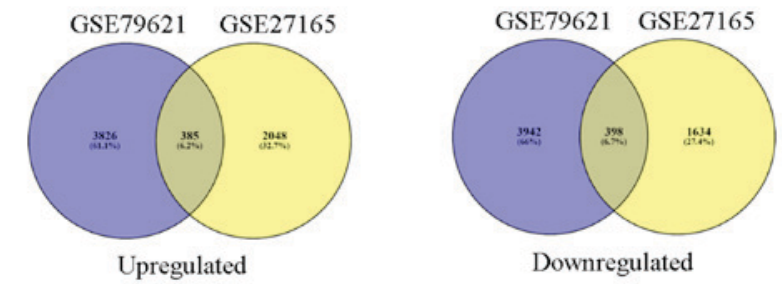

Figure 2. Co-expression of upregulated and downregulated genes in GSE79621 and GSE27165.

separately using Morpheus software. Then, a total of 4,211, 2,433 upregulated and 4,340,2,032 downregulated DEGs were identified from the GSE79621 and GSE27165 datasets, respectively. DEG expression heat map of the top 30 upregulated and downregulated genes of the two gene expression profiles are shown in Fig. 1. The 385 upregulated and 398 downregulated co-expressed genes were identified in the two gene expression profiles (Fig. 2).

GO term enrichment. The upregulated and downregulated DEGs were loaded to the DAVID software to identify GO categories and KEGG pathways. GO analysis results showed that in biological process, upregulated DEGs were significantly enriched in Arp2/3 complex-mediated actin nucleation, positive regulation of hyaluronan cable assembly, purine nucleobase biosynthetic process, de novo IMP biosynthetic process, and positive regulation of epithelial cell proliferation, whereas the downregulated DEGs were enriched in regulation of blood pressure, negative regulation of cell proliferation, ossification, negative regulation of gene expression and the type I interferon signaling pathway (Table I).
KEGG pathway analysis. The KEGG pathway analysis of upregulated and downregulated DEGs was performed using DAVID is presented in Table II. The top 5 KEGG pathways of upregulated DEGs were shigellosis, pathogenic Escherichia coli infection, mitogen-activated protein kinase (MAPK) signaling pathway, Ras signaling pathway and bacterial invasion of epithelial cells. The top 5 KEGG pathways of downregulated DEGs were systemic lupus erythematosus, lysosome, arachidonic acid metabolism, thyroid cancer and allograft rejection (Table II).

PPI network construction and module analysis. Based on the information in the STRING and Cytoscape databases, the top 10 hub nodes with high degrees were identified. These hub genes were GAPDH, MYC, CDK1, ACTB, APP, PRKACB, MAKP11, ACACB, HSPA4, CAT (Table III). GAPDH had the highest node degree, which was 83. A total of 6 modules from the PPI network satisfied the criteria of MCODE scores $\geq 4$ and number of nodes $>4$ (Table IV). The GO and KEGG pathway enrichment of the genes were included in the top module (Fig. 3) revealed that these genes were primarily associated with chloride transmembrane transport, chloride transport, positive regulation of cytosolic calcium ion concentration, chloride channel complex, chloride channel activity, GTP binding, extracellular-glycine-gated chloride channel activity, neuroactive ligand-receptor interaction, purine metabolism and intestinal immune network for IgA production (Table V).

\section{Discussion}

Despite advancements in the understanding of the mechanism of wound repair, an effective method for accelerating the 
Table II. KEGG pathway analysis of upregulated and downregulated differentially expressed genes. Top 5 terms were selected according to P-value when more than five terms enriched terms were identified in each category.

A, Upregulated

\begin{tabular}{|c|c|c|c|c|}
\hline Pathway ID & Name & Count & P-value & Genes \\
\hline hsa05131 & Shigellosis & 8 & $5.30 \times 10^{-4}$ & $\begin{array}{l}\text { ACTB, ARPC2, NFKBIB, ARPC5L, } \\
\text { ARPC4, MAPK11, MAPK10, FBXW11 }\end{array}$ \\
\hline hsa05130 & $\begin{array}{l}\text { Pathogenic Escherichia } \\
\text { coli infection }\end{array}$ & 6 & 0.005446999 & $\begin{array}{l}\text { ACTB, TUBB, ARPC } 2, \text { ARPC5L, } \\
\text { TUBA4A, ARPC4 }\end{array}$ \\
\hline hsa04010 & MAPK signaling pathway & 13 & 0.011844961 & $\begin{array}{l}\text { NTF3, MRAS, MAPK11, MAPK10, } \\
\text { ARRB2, RASGRP1, RASGRP2, } \\
\text { PRKACB, FGF1, MYC, GADD45A, } \\
\text { RASA1, NFATC1 }\end{array}$ \\
\hline hsa04014 & Ras signaling pathway & 12 & 0.012462871 & $\begin{array}{l}\text { LAT, GRIN2B, MRAS, HTR7, RASGRP1, } \\
\text { VEGFA, RASGRP2, GNB4, PRKACB, } \\
\text { MAPK10, FGF1, RASA1 }\end{array}$ \\
\hline hsa05100 & $\begin{array}{l}\text { Bacterial invasion of } \\
\text { epithelial cells }\end{array}$ & 6 & 0.030311184 & $\begin{array}{l}\text { ACTB, ARPC2, ARPC5L, ARPC4, CD2AP, } \\
\text { FN1 }\end{array}$ \\
\hline
\end{tabular}

B, Downregulated

\begin{tabular}{|c|c|c|c|c|}
\hline Pathway ID & Name & Count & P-value & Genes \\
\hline hsa05322 & $\begin{array}{l}\text { Systemic lupus } \\
\text { erythematosus }\end{array}$ & 10 & 0.006763183 & $\begin{array}{l}\text { HIST2H2AA3, HIST1H2AC, HIST1H2BD, } \\
\text { HIST1H2BK, HIST2H2BE, HIST2H2AC, } \\
\text { HLA-DPA1, C1R, C1S, CD } 40\end{array}$ \\
\hline hsa04142 & Lysosome & 9 & 0.011440506 & $\begin{array}{l}\text { CTSK, LAMP2, TPP1, GUSB, SMPD1, } \\
\text { PPT2, NEU1, ATP6V0A4, IDUA }\end{array}$ \\
\hline hsa00590 & $\begin{array}{l}\text { Arachidonic acid } \\
\text { metabolism }\end{array}$ & 6 & 0.019902025 & $\begin{array}{l}\text { PLA2G4A, TBXAS1, PTGS1, EPHX2, } \\
\text { LTA4H, PLA2G4C }\end{array}$ \\
\hline hsa05216 & Thyroid cancer & 4 & 0.036016354 & TCF7, RXRA, TFG, TCF7L1 \\
\hline hsa05330 & Allograft rejection & 4 & 0.066232041 & HLA-C, HLA-DPA1, HLA-B, CD40 \\
\hline
\end{tabular}

KEGG, Kyoto Encyclopedia of Genes and Genomes.

Table III. Degree of top 10 genes.

\begin{tabular}{llrl}
\hline Gene ID & \multicolumn{1}{c}{ Gene name } & Degree & Expression \\
\hline GAPDH & Glyceraldehyde-3-phosphate dehydrogenase & 83 & Upregulation \\
MYC & Myc proto-oncogene protein & 55 & Upregulation \\
CDK1 & Cyclin-dependent kinase 1 & 51 & Downregulation \\
ACTB & Actin, cytoplasmic 1 & 50 & Upregulation \\
APP & Amyloid beta A4 protein & 40 & Downregulation \\
PRKACB & cAMP-dependent protein kinase catalytic subunit beta & 36 & Upregulation \\
MAKP11 & Mitogen-activated protein kinase 11 & 35 & Upregulation \\
ACACB & Acetyl-CoA carboxylase 2 & 34 & Downregulation \\
HSPA4 & Heat shock 70 kDa protein 4 & 31 & Upregulation \\
CAT & Catalase & 31 & Downregulation \\
\hline
\end{tabular}

process remains to be identified. Investigation of the complicated molecular mechanism of wound repair is of important for treatment, particularly of chronic traumatic wounds, diabetic foot and bedsores. Previous studies $(31,32)$ have focused on the 
Table IV. Six modules from the protein-protein interaction network satisfied the criteria of MCODE scores $\geq 4$ and number of nodes $>4$.

\begin{tabular}{|c|c|c|c|c|}
\hline Cluster & Score & Nodes & Edges & Node IDs \\
\hline 1 & 6.353 & 35 & 108 & $\begin{array}{l}\text { FXYD1, ANO1, FXYD3, CD34, POLR3A, GCH1, GNA14, CCR2, NME4, } \\
\text { EDNRA, NME2, GUCY1A3, GUCY1A2, PTGER3, PROKR1, HDAC9, } \\
\text { TNFSF13B, TUBA4A, DNAJC10, CCT3, GLRB, GLRA1, IARS, CLIC4, } \\
\text { CXCL12, CXCR4, RAD51C, GRM7, F2R, RAD51, GAPDH, MKKS, APP, } \\
\text { SAA1, ATF3 }\end{array}$ \\
\hline 2 & 4.8 & 6 & 12 & MRAS, LRRC2, FMOD, RAP2C, MST4, PDE7B \\
\hline 3 & 4.5 & 9 & 18 & HLA-B, IRF4, GLDC, ACACB, OAS3, GART, PMPCB, IFIT3, HLA-C \\
\hline 4 & 4.5 & 5 & 9 & WDR4, CECR1, WDR12, PUS1, PUS7 \\
\hline 5 & 4.3 & 21 & 43 & $\begin{array}{l}\text { MYC, DHX9, MDM2, MCL1, VAMP1, YWHAG, RABL6, UPF2, STX16, TUBB, } \\
\text { NUDT21, RAB6A, NSF, RPL37, RPL22, TUBB4Q, PHF5A, ACTB, VAMP4, } \\
\text { STX6, AR }\end{array}$ \\
\hline 6 & 4 & 4 & 6 & COL6A2, COL13A1, COL4A4, COL11A1 \\
\hline
\end{tabular}

Score $=($ Density $\mathrm{x}$ no. of nodes $)$.

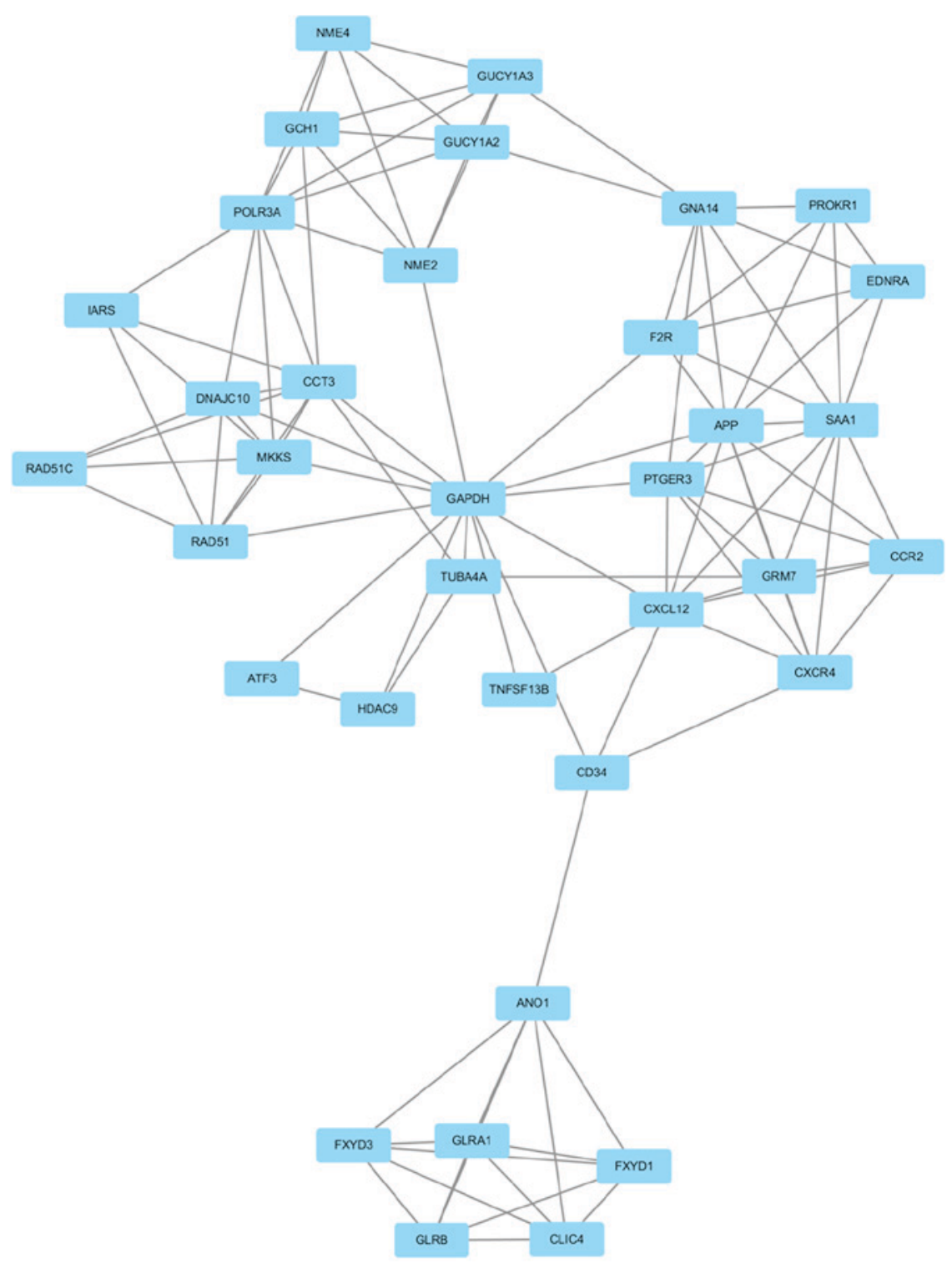

Figure 3. Top module from the protein-protein interaction network. 
Table V. Functional and pathway enrichment analysis of the genes in module. Top 3 terms were selected according to P-value when more than 3 terms enriched terms were identified in each category.

A, Biological processes

\begin{tabular}{llccl}
\hline Term & \multicolumn{1}{c}{ Name } & Count & P-value & \multicolumn{1}{c}{ Genes } \\
\hline GO:1902476 & $\begin{array}{l}\text { Chloride transmembrane } \\
\text { transport } \\
\text { Chloride transport }\end{array}$ & 6 & $1.15 \times 10^{-6}$ & $\begin{array}{l}\text { FXYD1, GLRB, FXYD3, } \\
\text { GLRA1, CLIC4, ANO1 }\end{array}$ \\
GO:0006821 & $\begin{array}{l}\text { Positive regulation of cytosolic } \\
\text { calcium ion concentration }\end{array}$ & 6 & $1.22 \times 10^{-6}$ & $\begin{array}{l}\text { FXYD1, FXYD3, GLRA1, } \\
\text { CLIC4, ANO1 }\end{array}$ \\
GO:0007204 & E.60x 10-6 & EDNRA, PTGER3, CXCR4, \\
& & & SAA1, CCR2, F2R
\end{tabular}

B, Molecular functions

\begin{tabular}{llccc}
\hline Term & \multicolumn{1}{c}{ Name } & Count & P-value & Genes \\
\hline GO:0005254 & Chloride channel activity & 4 & $2.02 \times 10^{-4}$ & FXYD1, FXYD3, CLIC4, ANO1 \\
GO:0005525 & GTP binding & 5 & 0.007953298 & $\begin{array}{l}\text { GNA14, GUCY1A2, GUCY1A3, } \\
\text { TUBA4A, GCH1 } \\
\text { GO:0016934 }\end{array}$ \\
& $\begin{array}{l}\text { Extracellular-glycine-gated } \\
\text { chloride channel activity }\end{array}$ & 2 & 0.010379054 & GLRB, GLRA1
\end{tabular}

C, KEGG pathways

\begin{tabular}{llccl}
\hline Term & \multicolumn{1}{c}{ Name } & Count & P-value & \multicolumn{1}{c}{ Genes } \\
\hline hsa04080 & $\begin{array}{l}\text { Neuroactive ligand-receptor } \\
\text { interaction } \\
\text { Purine metabolism }\end{array}$ & 6 & 0.003899897 & $\begin{array}{l}\text { EDNRA, GLRB, PTGER3, } \\
\text { GLRA1, GRM7, F2R } \\
\text { hsa00230 }\end{array}$ \\
hsa04672 & $\begin{array}{l}\text { Intestinal immune network for } \\
\text { IgA production }\end{array}$ & 3 & 0.014264846 & $\begin{array}{l}\text { GUCY1A3, POLR3A } \\
\text { TNFSF13B, CXCR4, CXCL12 }\end{array}$ \\
& & &
\end{tabular}

KEGG, Kyoto Encyclopedia of Genes and Genomes.

effect of vascular endothelial cells, epithelial cells and bone marrow mesenchymal stem cells in wound and the effect of fibroblasts remains to be determined. The proliferation phase ensues with fibroblast migration, which then synthesize ECM components, which have a significant role in each stage of the healing process. TGF- $\beta$ contributes to the function of fibroblasts, including cell migration, collagen synthesis and cell proliferation. Therefore, understanding the effect of TGF- $\beta$ on fibroblasts at the early proliferation stage is essential to identify an effective way to improve wound healing. The present study used bioinformatics analysis to investigate the effect of TGF- $\beta$ on fibroblasts at the early proliferation stage of wound healing. Data from GSE79621 and GSE27165 was obtained and identified 385 upregulated and 398 downregulated overlapped DEGs between normal fibroblasts and TGF- $\beta 24 \mathrm{~h}$ treated fibroblasts. In order to understand the interactions of DEGs, GO and KEGG pathway analyses were performed.

The GO term analysis showed that the upregulated DEGs were mainly involved in Arp2/3 complex-mediated actin nucleation, positive regulation of hyaluronan cable assembly, purine nucleobase biosynthetic process, de novo IMP biosynthetic process and positive regulation of epithelial cell proliferation. Previous studies have found that actin has an important role in cell migration (33-35). The GO term analysis in biological processes suggested that at the proliferation phase, TGF- $\beta$ may regulate actin-based fibroblast migration to the wound site by Arp2/3 complex. Previous studies have demonstrated that fetal wounds consist of ECM with an abundance of hyaluronan $(36,37)$. Hyaluronan is produced by fibroblasts and promotes fibroblasts migration and proliferation early in the repair process (37). Therefore, TGF- $\beta$-mediated hyaluronan synthesis may be essential for the migration and proliferation of fetal fibroblasts. In addition, the findings of the present study also suggest that TGF- $\beta$ may regulate the biological activity of fibroblasts, purine nucleobase biosynthetic process and de novo IMP biosynthetic process. Furthermore, TGF- $\beta$ is the only growth factor, which accelerates 'maturation' of epithelial cell layers (38), which is accordance with the current findings 
that TGF- $\beta$ is related to the positive regulation of epithelial cell proliferation. The GO term analysis revealed that the downregulated DEGs were primarily involved in the regulation of blood pressure, negative regulation of cell proliferation, ossification, gene expression and the type I interferon signaling pathway. The regulation of blood pressure is a process controlled by a balance of processes that increase pressure and reduce pressure. A previous study determined that blood pressure is negatively regulated by TGF- $\beta$ (39), which was consistent the current findings. Ossification is the conversion of fibrous tissue into bone or a bony substance. Inhibition of ossification by downregulation of genes (Bone morphogenic protein 1, Ras association domain-containing protein 2 , neuronal membrane glycoprotein M6-b, atrial natriuretic peptide receptor 2, exostosin-1, V-type proton ATPase $116 \mathrm{kDa}$ subunit a isoform 4 and extracellular matrix protein1) may imply that the early stage of wound healing is mainly associated with fibroblast proliferation and collagen synthesis. Increased levels of genes in the type I interferon pathway have been observed in dermal fibrosis (40). The regulation mechanism of type I interferon of dermal fibroblasts and its participation in the development of dermal fibrosis remains to be elucidated. The current findings suggest that TGF- $\beta$ may inhibit the interferon signaling pathway of fibroblasts by downregulation of gene expression levels, thus preventing wound fibrosis at early stage. Previous studies have found that TGF- $\beta$ could mediate cell proliferation $(41,42)$ and collagen production $(43,44)$. The GO term analysis demonstrated that downregulated genes were mainly enriched in negative regulation of cell proliferation and gene expression, suggesting that TGF- $\beta$ could promote fibroblast proliferation and collagen synthesis at the early stage of wound healing by downregulating related genes. In addition, the enriched KEGG pathways of upregulated DEGs included shigellosis, pathogenic Escherichia coli infection, MAPK signaling pathway, Ras signaling pathway and bacterial invasion of epithelial cells. The pathway of shigellosis, pathogenic Escherichia coli infection and bacterial invasion of epithelial cells was associated with infection, suggesting that TGF- $\beta$ has an antibacterial effect on the healing of infected wounds. Previous studies have demonstrated that activation the MAPK and Ras signaling pathways could promote cell proliferation, differentiation and migration $(45,46)$. In addition, the top 5 KEGG pathways of the downregulated DEGs were systemic lupus erythematosus, lysosome, arachidonic acid metabolism, thyroid cancer and allograft rejection. The systemic lupus erythematosus pathway and allograft rejection pathways are associated with autoantibodies and could cause tissue injury. Lysosomes serve as the cell's main digestive compartment and macromolecules are delivered for degradation. However, previous studies have found (47) that ingested microparticles may induce cellular damage in phagocytes through the release of lysosomal enzymes following lysosome rupture. Arachidonic acid is oxygenated and further transformed into a variety of products, which mediate inflammatory reactions (48).

A PPI network was constructed from the DEGs by Cytoscape and the 10 genes exhibiting the highest degree of connectivity. From these 10 genes, 6 genes were upregulated (GAPDH, MYC, ACTB, PRKACB, MAKP11, HSPA4) and 4 genes (CDK1, APP, ACACB, CAT) were downregulated. The top hub gene GAPDH, encodes a member of the glyceraldehyde-3-phosphate dehydrogenase protein family, which catalyzes an important energy-yielding step in carbohydrate metabolism. A previous study has determined that GAPDH could maintain the integrity of a protein (49). Lin et al (50) reported that MYC plays a positive role in regulation of fibroblasts proliferation. ACTB encodes one of actin proteins, which are highly conserved proteins involved in various types of cell motility. A previous study determined that $\beta$-actin has a key role in cell growth and migration (51). The protein encoded by PRKACB is a member of the serine/threonine protein kinase family. The encoded protein is a catalytic subunit of cAMP-dependent protein kinase, which mediates signaling though cAMP. cAMP signaling is important for various processes, including cell proliferation and differentiation (52). MAKP11 encodes mitogen-activated protein kinase 11 , which is a member of the protein kinases family. MAKP11 is involved in the integration of biochemical signals for a wide variety of cellular processes, including cell proliferation, differentiation, transcriptional regulation and development $(53,54)$. The activity of the heat shock protein A4 (HSPA4), a member of the HSP110 family, is inducible under various conditions (55). However, a previous study revealed that overexpression of HSPA4 may inhibit the migration of fibroblasts cells (56). The 4 downregulated hub genes were CDK1, APP, ACACB and CAT. CDK1, also termed CDC2, has a key role in the control of cell cycles and promotes cell migration and proliferation $(49,57,58)$. APP encodes amyloid $\beta$ A4 protein, an integral membrane protein largely known for its role as the precursor of A $\beta$ peptides and for its involvement in the pathogenesis of Alzheimer's disease (59). Previous studies have determined that SAPP, the secretory domain of the APP has been observed to reestablish cell growth in APP-deficient fibroblasts (60). Acetyl-CoA carboxylase $\beta$ is encoded by ACACB regulates cellular metabolic processes and may be involved in the regulation of fatty acid oxidation. CAT encodes catalase, a key antioxidant enzyme in the defense against oxidative stress. Downregulation of ACACB and CAT may promote wound repair through prevention of oxidative stress (61). It is of note that at the early proliferation stage of wound repair, fibroblasts migrate to the wound site from surrounding tissue, and contribute to the proliferation and synthesis ECM components. However, the current study revealed that TGF- $\beta$ upregulated the expression level of HSPA4 and downregulated the level of CDK1 at the early wound healing stage. It is of note that previous studies determined that overexpression of HSPA4 and reduced expression of CDK1 may inhibit cell migration and proliferation $(56,58)$. Therefore, TGF- $\beta$ may inhibit excessive fibroblast migration and proliferation by meditating the expression level of different genes. Therefore, further investigation is required in order to clarify the underlying biological mechanisms of HSPA4 and CDK1 on fibroblasts at the early wound healing stage.

A previous study revealed that ion channels and transporters have a key role in cellular functions (62). Their physiological roles in cell proliferation have been considered, as cell volume changes, which involves the movement of ions across the cell membrane, which is essential for cell-cycle progression $(63,64)$. The biological process and molecular function analysis identified one module that contained genes mainly enriched in chloride and calcium channels, which also suggested that ions 
are important in fibroblast migration and proliferation. However, the mechanism behind this the regulation of this process by ion channels is still unclear. Therefore, future studies should investigate the genes associated with ion channels to elucidate how TGF- $\beta$ mediated ion channels and in turn cellular functions. Furthermore, the pathway analysis revealed that the effect of TGF- $\beta$ on fibroblasts was associated with neuroactive ligand-receptor interaction, purine metabolism and intestinal immune network for IgA production. A previous study determined that fibroblasts could interact with other cells during the process of wound repair (65). It is of note that, tenascin-C derived from fibroblasts could promote Schwann cell migration to the wound site and peripheral nerve regeneration (66). It has been established that purine metabolites provide a cell with the necessary energy to promote cell progression $(56,67)$. One feature of intestinal immunity is its ability to generate non-inflammatory immunoglobulin A antibodies that act as the first line of defense against microorganisms. The aforementioned GO and KEGG pathway analyses of the top module suggest that TGF- $\beta$ could promote fibroblast proliferation, migration and have an antimicrobial effect.

The current study has several limitations. Firstly, the sample size was small and samples were not directly obtained from the wound. Wound repair is a complex interaction process and except TGF- $\beta$, other cytokines also have effect on fibroblasts. Secondly, the proliferation stage may continue for 3-10 days and TGF- $\beta$ may regulate other genes included in fibroblasts. Thirdly, these finding would benefit from a validation by western blotting and polymerase chain reaction. However, the present findings may suggest potential methods to investigate the effect of TGF- $\beta$ on fibroblasts. In addition, future studies will be designed to clarify the role of TGF- $\beta$ on fibroblasts during the early stage of wound repair.

In conclusion, the current study provided a comprehensive bioinformatics analysis of DEGs, which was associated with the effect of TGF- $\beta$ on fibroblasts at the early proliferation phase of wound repair. The current study provided a set of useful target genes and pathways for further investigation into the molecular mechanisms of wound repair. In addition, the current study may provide a novel research method, which is rarely used in wound repair. Additional clinical samples are required to confirm the DEGs of fibroblasts affected by TGF- $\beta$ during wound repair.

\section{Acknowledgements}

The authors would like to thank Mr Liming Xiong for the support and the design idea for the current study.

\section{References}

1. Ueha S, Shand FH and Matsushima K: Cellular and molecular mechanisms of chronic inflammation-associated organ fibrosis. Front Immunol 3: 71, 2012.

2. Jacinto A, Martinez-Arias A and Martin P: Mechanisms of epithelial fusion and repair. Nat Cell Biol 3: E117-E123, 2001.

3. Baer ML and Colello RJ: Endogenous bioelectric fields: A putative regulator of wound repair and regeneration in the central nervous system. Neural Regen Res 11: 861-864, 2016.

4. Xie SY, Peng LH, Shan YH, Niu J, Xiong J and Gao JQ: Adult stem cells seeded on electrospinning silk fibroin nanofiberous scaffold enhance wound repair and regeneration. J Nanosci Nanotechnol 16: 5498-5505, 2016.
5. Smith-Bolton R: Drosophila imaginal discs as a model of epithelial wound repair and regeneration. Adv Wound Care (New Rochelle) 5: 251-261, 2016.

6. Yang S, Ma K, Geng Z, Sun X and Fu X: Oriented cell division: New roles in guiding skin wound repair and regeneration. Biosci Rep 35: pii: e00280, 2015.

7. Peng LH, Wei W, Shan YH, Zhang TY, Zhang CZ, Wu JH, Yu L, Lin J, Liang WQ, Khang G and Gao JQ: $\beta$-Cyclodextrin-linked polyethylenimine nanoparticles facilitate gene transfer and enhance the angiogenic capacity of mesenchymal stem cells for wound repair and regeneration. J Biomed Nanotechnol 11: 680-690, 2015.

8. Kotwal GJ, Sarojini H and Chien S: Pivotal role of ATP in macrophages fast tracking wound repair and regeneration. Wound Repair Regen 23: 724-727, 2015.

9. Shingyochi Y, Orbay H and Mizuno H: Adipose-derived stem cells for wound repair and regeneration. Expert Opin Biol Ther 15: 1285-1292, 2015 .

10. Eming SA, Martin P and Tomic-Canic M: Wound repair and regeneration: Mechanisms, signaling, and translation. Sci Transl Med 6: 265sr6, 2014.

11. Xu S, Sang L, Zhang Y, Wang X and Li X: Biological evaluation of human hair keratin scaffolds for skin wound repair and regeneration. Mater Sci Eng C Mater Biol Appl 33: 648-655, 2013.

12. Sen CK and Roy S: OxymiRs in cutaneous development, wound repair and regeneration. Semin Cell Dev Biol 23: 971-980, 2012.

13. Reinke JM and Sorg H: Wound repair and regeneration. Eur Surg Res 49: 35-43, 2012.

14. Li H and Fu X: Mechanisms of action of mesenchymal stem cells in cutaneous wound repair and regeneration. Cell Tissue Res 348: 371-377, 2012.

15. Eldardiri M, Martin Y, Roxburgh J, Lawrence-Watt DJ and Sharpe JR: Wound contraction is significantly reduced by the use of microcarriers to deliver keratinocytes and fibroblasts in an in vivo pig model of wound repair and regeneration. Tissue Eng Part A 18: 587-597, 2012.

16. PengLH,Tsang SY,Tabata Y and GaoJQ: Genetically-manipulated adult stem cells as therapeutic agents and gene delivery vehicle for wound repair and regeneration. J Control Release 157: 321-330, 2012.

17. Fu X and Li H: Mesenchymal stem cells and skin wound repair and regeneration: Possibilities and questions. Cell Tissue Res 335: 317-321, 2009.

18. Gurtner GC, Werner S, Barrandon Y and Longaker MT: Wound repair and regeneration. Nature 453: 314-321, 2008.

19. Yao F and Eriksson E: Gene therapy in wound repair and regeneration. Wound Repair Regen 8: 443-451, 2000.

20. Puchelle E: Airway epithelium wound repair and regeneration after injury. Acta Otorhinolaryngol Belg 54: 263-270, 2000.

21. Clark LD, Clark RK and Heber-Katz E: A new murine model for mammalian wound repair and regeneration. Clin Immunol Immunopathol 88: 35-45, 1998.

22. Sicard RE and Nguyen LM: An in vivo model for evaluating wound repair and regeneration microenvironments. In Vivo 10: 477-481, 1996.

23. Suga H, Sugaya M, Fujita H, Asano Y, Tada Y, Kadono T and Sato S: TLR4, rather than TLR2, regulates wound healing through TGF- $\beta$ and CCL5 expression. J Dermatol Sci 73: 117-124, 2014.

24. Lee MJ, Shin JO and Jung HS: Thy-1 knockdown retards wound repair in mouse skin. J Dermatol Sci 69: 95-104, 2013.

25. Liu J, Wang Y, Pan Q, Su Y, Zhang Z, Han J, Zhu X, Tang C and $\mathrm{Hu} \mathrm{D}$ : Wnt $/ \beta$-catenin pathway forms a negative feedback loop during TGF- $\beta 1$ induced human normal skin fibroblast-to-myofibroblast transition. J Dermatol Sci 65: 38-49, 2012.

26. Chen J, Zeng B, Yao H and Xu J: The effect of TLR4/7 on the TGF- $\beta$-induced Smad signal transduction pathway in human keloid. Burns 39: 465-472, 2013.

27. Diegelmann RF and Evans MC: Wound healing: An overview of acute, fibrotic and delayed healing. Front Biosci 9: 283-289, 2004.

28. Elgaaen BV, Olstad OK, Sandvik L, Odegaard E, Sauer T, Staff AC and Gautvik KM: ZNF385B and VEGFA are strongly differentially expressed in serous ovarian carcinomas and correlate with survival. PLoS One 7: e46317, 2012.

29. Li C, Zhen G, Chai Y, Xie L, Crane JL, Farber E, Farber CR, Luo X, Gao P, Cao X and Wan M: RhoA determines lineage fate of mesenchymal stem cells by modulating CTGF-VEGF complex in extracellular matrix. Nat Commun 7: 11455, 2016. 
30. Al-Rekabi Z, Wheeler MM, Leonard A, Fura AM, Juhlin I, Frazar C, Smith JD, Park SS, Gustafson JA, Clarke CM, et al: Activation of the IGF1 pathway mediates changes in cellular contractility and motility in single-suture craniosynostosis. J Cell Sci 129: 483-491, 2016.

31. Chigurupati S, Mughal MR, Okun E, Das S, Kumar A, McCaffery M, Seal S and Mattson MP: Effects of cerium oxide nanoparticles on the growth of keratinocytes, fibroblasts and vascular endothelias cells in cutaneous wound healing. Biomaterials 34: 2194-2201, 2013.

32. Zhao H, Han T, Hong $X$ and Sun D: Adipose differentiation-related protein knockdown inhibits vascular smooth muscle cell proliferation and migration and attenuates neointima formation. Mol Med Rep 16: 3079-3086, 2017.

33. Ridley AJ, Schwartz MA, Burridge K, Firtel RA, Ginsberg MH, Borisy G, Parsons JT and Horwitz AR: Cell migration: Integrating signals from front to back. Science 302: 1704-1709, 2003.

34. Le Clainche $\mathrm{C}$ and Carlier MF: Regulation of actin assembly associated with protrusion and adhesion in cell migration. Physiol Rev 88: 489-513, 2008.

35. Fotedar R and Margolis RL. WISp39 and Hsp90: Actin' together in cell migration. Oncotarget 6: 17871-17872, 2015.

36. Mast BA, Diegelmann RF, Krummel TM and Cohen IK: Hyaluronic acid modulates proliferation, collagen and protein synthesis of cultured fetal fibroblasts. Matrix 13: 441-446, 1993.

37. Balaji S, King A, Marsh E, LeSaint M, Bhattacharya SS, Han N, Dhamija Y, Ranjan R, Le LD, Bollyky PL, et al: The role of interleukin-10 and hyaluronan in murine fetal fibroblast function in vitro: Implications for recapitulating fetal regenerative wound healing. PLoS One 10: e124302, 2015.

38. Wu YS and Chen SN: Apoptotic cell: Linkage of inflammation and wound healing. Front Pharmacol 5: 1, 2014.

39. Matsuki K, Hathaway CK, Lawrence MG, Smithies O and Kakoki M: The role of transforming growth factor $\beta 1$ in the regulation of blood pressure. Curr Hypertens Rev 10: 223-238, 2014.

40. Agarwal SK, Wu M, Livingston CK, Parks DH, Mayes MD, Arnett FC and Tan FK: Toll-like receptor 3 upregulation by type I interferon in healthy and scleroderma dermal fibroblasts. Arthritis Res Ther 13: R3, 2011.

41. Zhang Y, Alexander PB and Wang XF: TGF- $\beta$ family signaling in the control of cell proliferation and survival. Cold Spring Harb Perspect Biol 9: pii: a022145, 2017.

42. DiRenzo DM, Chaudhary MA, Shi X, Franco SR, Zent J, Wang K, Guo LW and Kent KC: A crosstalk between TGF- $\beta /$ Smad 3 and Wnt/beta-catenin pathways promotes vascular smooth muscle cell proliferation. Cell Signal 28: 498-505, 2016.

43. Ghosh AK, Mori Y, Dowling E and Varga J: Trichostatin A blocks TGF-beta-induced collagen gene expression in skin fibroblasts: Involvement of Sp1. Biochem Biophys Res Commun 354 420-426, 2007.

44. Lu J, Shi J, Li M, Gui B, Fu R, Yao G, Duan Z, Lv Z, Yang Y, Chen Z, et al: Activation of AMPK by metformin inhibits TGF- $\beta$-induced collagen production in mouse renal fibroblasts. Life Sci 127: 59-65, 2015 .

45. Zhang W and Liu HT: MAPK signal pathways in the regulation of cell proliferation in mammalian cells. Cell Res 12: 9-18, 2002

46. Takino K, Ohsawa S and Igaki T: Loss of Rab5 drives non-autonomous cell proliferation through TNF and Ras signaling in Drosophila. Dev Biol 395: 19-28, 2014

47. Gause WC, Wynn TA and Allen JE: Type 2 immunity and wound healing: Evolutionary refinement of adaptive immunity by helminths. Nat Rev Immunol 13: 607-614, 2013.

48. Samuelsson B: Arachidonic acid metabolism: Role in inflammation. Z Rheumatol 50 (Suppl 1): S3-S6, 1991.
49. Chen X, Stauffer S, Chen Y and Dong J: Ajuba Phosphorylation by CDK1 Promotes Cell Proliferation and Tumorigenesis. J Biol Chem 291: 14761-14772, 2016.

50. Lin L, Zhang JH, Panicker LM and Simonds WF: The parafibromin tumor suppressor protein inhibits cell proliferation by repression of the c-myc proto-oncogene. Proc Natl Acad Sci USA 105: 17420-17425, 2008

51. Bunnell TM, Burbach BJ, Shimizu Y and Ervasti JM: $\beta$-Actin specifically controls cell growth, migration, and the G-actin pool. Mol Biol Cell 22: 4047-4058, 2011.

52. Liu D, Huang Y, Bu D, Liu AD, Holmberg L, Jia Y, Tang C, Du J and Jin H: Sulfur dioxide inhibits vascular smooth muscle cell proliferation via suppressing the Erk/MAP kinase pathway mediated by cAMP/PKA signaling. Cell Death Dis 5: e1251, 2014.

53. Jiang Y, Chen C, Li Z, Guo W, Gegner JA, Lin S and Han J: Characterization of the structure and function of a new mitogen-activated protein kinase (p38beta). J Biol Chem 271: 17920-17926, 1996.

54. Hsu YL, Wang MY, Ho LJ, Huang CY and Lai JH: Up-regulation of galectin-9 induces cell migration in human dendritic cells infected with dengue virus. J Cell Mol Med 19: 1065-1076, 2015.

55. Adachi T, Sakurai T, Kashida H, Mine H, Hagiwara S, Matsui S, Yoshida K, Nishida N, Watanabe T, Itoh K, et al: Involvement of heat shock protein a4/apg-2 in refractory inflammatory bowel disease. Inflamm Bowel Dis 21: 31-39, 2015.

56. Sakurai T, Kashida H, Hagiwara S, Nishida N, Watanabe T, Fujita J and Kudo M: Heat shock protein A4 controls cell migration and gastric ulcer healing. Dig Dis Sci 60: 850-857, 2015.

57. Wang Z, Fan M, Candas D, Zhang TQ, Qin L, Eldridge A, Wachsmann-Hogiu S, Ahmed KM, Chromy BA, Nantajit D, et al: Cyclin B1/Cdk1 coordinates mitochondrial respiration for cell-cycle G2/M progression. Dev Cell 29: 217-232, 2014.

58. Han IS, Seo TB, Kim KH, Yoon JH, Yoon SJ and Namgung U: Cdc2-mediated Schwann cell migration during peripheral nerve regeneration. J Cell Sci 120: 246-255, 2007.

59. Dawkins E and Small DH. Insights into the physiological function of the $\beta$-amyloid precursor protein: Beyond Alzheimer's disease. J Neurochem 129: 756-769, 2014

60. Saitoh T, Sundsmo M, Roch JM, Kimura N, Cole G, Schubert D, Oltersdorf T and Schenk DB: Secreted form of amyloid beta protein precursor is involved in the growth regulation of fibroblasts. Cell 58: 615-622, 1989 .

61. Ponugoti B, Xu F, Zhang C, Tian C, Pacios S and Graves DT: FOXO1 promotes wound healing through the up-regulation of TGF- $\beta 1$ and prevention of oxidative stress. J Cell Biol 203: 327-343, 2013.

62. Wang YL, Sun GY, Zhang Y, He JJ, Zheng S and Lin JN: Tormentic acid inhibits $\mathrm{H} 2 \mathrm{O} 2$-induced oxidative stress and inflammation in rat vascular smooth muscle cells via inhibition of NF-kB signaling pathway. Mol Med Rep 14: 3559-3564, 2016.

63. Ohsawa R, Miyazaki H, Niisato N, Shiozaki A, Iwasaki Y, Otsuji E and Marunaka Y: Intracellular chloride regulates cell proliferation through the activation of stress-activated protein kinases in MKN28 human gastric cancer cells. J Cell Physiol 223: 764-770, 2010.

64. Bear CE: Phosphorylation-activated chloride channels in human skin fibroblasts. Febs Lett 237: 145-149, 1988.

65. Sorrell JM and Caplan AI: Fibroblasts-a diverse population at the center of it all. Int Rev Cell Mol Biol 276: 161-214, 2009.

66. Zhang Z, Yu B, Gu Y, Zhou S, Qian T, Wang Y, Ding G, Ding F and Gu X: Fibroblast-derived tenascin-C promotes Schwann cell migration through $\beta 1$-integrin dependent pathway during peripheral nerve regeneration. Glia 64: 374-385, 2016

67. Pedley AM and Benkovic SJ: A new view into the regulation of purine metabolism: The purinosome. Trends Biochem Sci 42: 141-154, 2017. 